\title{
Analyse des propriétés sensorielles des ignames cultivées à Madagascar*
}

\author{
Boris Ranaivosoa ${ }^{1}$ \\ Dominique Valentin ${ }^{2}$ \\ Vololoniaina Harimanga Jeannoda ${ }^{3}$ \\ Julia Louisette Razanamparany ${ }^{1}$ \\ ${ }^{1}$ Université d'Antananarivo \\ Laboratoire de biochimie appliquée \\ aux sciences de l'alimentation (LABASAN) \\ BP 906 \\ Antananarivo 101 \\ Madagascar \\ <ranaivosoaboris@yahoo.fr> \\ <razanamparany.louisette@yahoo.fr> \\ 2 Université de Bourgogne \\ CSGA \\ 15, rue Hugues Picardet \\ 21000 Dijon \\ France \\ <valentin@u-bourgogne.fr> \\ ${ }^{3}$ Université d'Antananarivo \\ Faculté des sciences \\ Département de biologie et écologie végétales \\ Antananarivo \\ Madagascar \\ <vololoniaina.jeannoda@gmail.com>
}

\begin{abstract}
Résumé
Les propriétés organoleptiques des tubercules d'igname appartenant à l'espèce Dioscorea esculenta, et trois formes de Dioscorea alata L. (ovibe, ovilalaina, ovifotsy), les plus cultivées de Madagascar, ont été déterminées dans cette étude. Un test hédonique a révélé un effet significatif de l'origine et de l'âge des consommateurs sur l'appréciation globale des tubercules d'ignames. Quel que soit leur âge, les consommateurs des régions productrices d'ignames les apprécient davantage que les consommateurs issus des régions non productrices. Dans ces régions non productrices, les adultes apprécient davantage les ignames que les enfants d'âge scolaire. En dépit de ces différences globales d'appréciation, l'ordre de préférence des variétés d'ignames a été le même pour tous les groupes de consommateurs : D. esculenta et la forme ovilalaina, sont les plus appréciées. Un test descriptif et des mesures physico-chimiques ont mis en évidence que les tubercules D. esculenta et la forme ovilalaina comportent davantage de glucides, d'amidon, de calories, et sont perçues comme étant plus sucrées et plus faciles à avaler. Le caractère sucré des ignames est recherché en particulier par les enfants issus des régions non productrices.
\end{abstract}

Mots clés : consommateur ; igname ; Madagascar ; propriété physicochimique ; test sensoriel.

Thèmes : productions végétales ; qualité et sécurité des produits.

\section{Abstract \\ Sensory property analysis of yams cultivated in Madagascar}

The potential development of the four varieties of yam the most commonly cultivated in Madagascar, Dioscorea esculenta (Lour), and Dioscorea alata L. cv ovibe, ovilalaina, ovifotsy were determined in this study. A hedonic test showed the main effect of consumer origin and age as well as an interaction between the two factors. Regardless of age, consumers from yam regions preferred yams more than did consumers from regions where yams are not cultivated. In the latter regions, adult consumers preferred yams more than did children. Besides these differences in global appreciation of yams, D. esculenta (Lour) and the ovilalaina cultivar obtained the highest preference scores from all consumers. A descriptive test and physicochemical measures indicate that D. esculenta (Lour) and the ovilalaina cultivar contain more carbohydrates, starch and calories and are described as sweet and easy to swallow. Sweetness seems to be particularly sought by children from regions where yams are not cultivated.

Key words: chemicophysical properties; consumers; Madagascar; sensory testing; yam. Subjects: vegetal productions; product quality and security. 
Madagascar, le genre Dioscorea comporte plus de 40 espèces, soit environ le dixième des 450 espèces d'ignames actuellement reconnues dans le monde, selon les dernières mises au point phylogénétiques (Perrier de la Bâthie, 1925 ; Wilkin et al., 2005). Pour améliorer la disponibilité et les possibilités d'utilisation des tubercules d'ignames, plusieurs études ont été effectuées, notamment sur les ignames domestiquées $D$. cayenensis Lam., D. rotundata Poir. et $D$. dumetorum d'Afrique occidentale et $D$. alata L, espèce introduite et provenant d'Asie. Ces études portaient sur l'amélioration du potentiel agronomique de ces variétés d'ignames (Vernier et al., 2000 ; Vernier et Dossou, 2003 ; Dumont et al., 2005), sur la caractérisation physico-chimique et les propriétés fonctionnelles des amidons (Trèche et al., 1984 ; Onayemi et Idowu, 1988 ; Amani, 2002 ; Razanamparany et al., 2003 ; Dumont et al., 2005), la transformation de ces ignames en cossettes, les perspectives de valorisation alimentaire couplées à la méthode d'évaluation sensorielle (Onayemi, 1985; Vernier et al., 2000 ; Akissoe et al., 2001 ; Nindjin et al., 2002;
Brunnschweiler et al., 2005 ; Nindjin et al., 2006 ). Concernant les ignames cultivées à Madagascar, des études ont été menées sur la diversité écologique, l'utilisation traditionnelle et le système de gestion durable. Ces études ont montré une grande diversité des cultivars dans le versant oriental, humide, et le versant occidental, sec, de Madagascar. L'espèce $D$. esculenta (Lour) (figure 1A), et les formes de l'espèce $D$. alata (ovibe, ovilalaina, ovifotsy) (figures $1 B, 1 C, 1 D$ ), constituent les tubercules d'ignames les plus cultivés et se rencontrent surtout dans le versant oriental et humide de Madagascar (Jeannoda et al., 2003 ; Jeannoda et al., 2007). Dans cette région, la récolte se fait pendant la période de soudure (lorsque les réserves de riz sont épuisées), qui s'étend de mars à août, pendant la saison sèche.

L'objectif de cette étude est d'explorer les facteurs déterminant l'appréciation des ignames cultivées de Madagascar par des consommateurs habituels et potentiels. Une meilleure compréhension de ces facteurs permettrait de développer l'appréciation et la consommation des ignames et ainsi d'apporter une diversification ali- mentaire dans un pays dont l'alimentation se caractérise par une grande monotonie. Nous avons réalisé en parallèle un test consommateur, un profil descriptif, et des mesures physico-chimiques des quatre variétés d'ignames les plus consommées à Madagascar. Le test consommateur visait à évaluer les préférences des consommateurs en fonction de leur âge et de leur origine géographique. Le profil sensoriel et la caractérisation physico-chimique ont ensuite été mis en relation avec les données de préférence pour mieux comprendre les facteurs fondant les préférences des consommateurs.

\section{Matériel et méthode}

\section{Localisation du site de collecte des tubercules}

La collecte des tubercules a été menée dans quelques régions de la côte est de Madagascar (figure 2 et tableau 1).
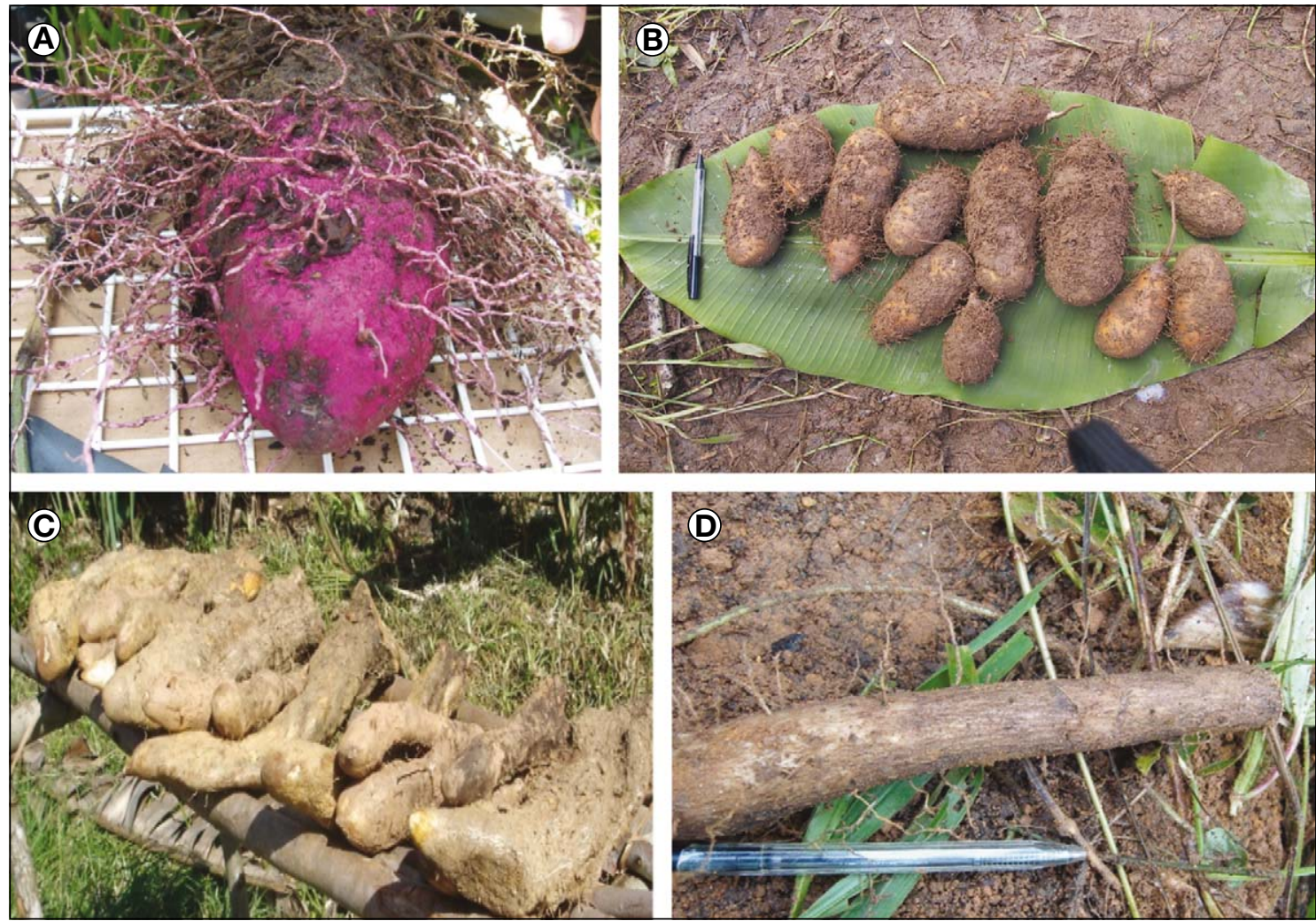

Figure 1. Tubercules d'ignames.

Figure 1. Yams tubers.

A) Dioscorea esculenta ; B) Dioscorea alata forme ovilalaina ; C) Dioscorea alata forme ovibe ; D) Dioscorea alata forme ovifotsy. 


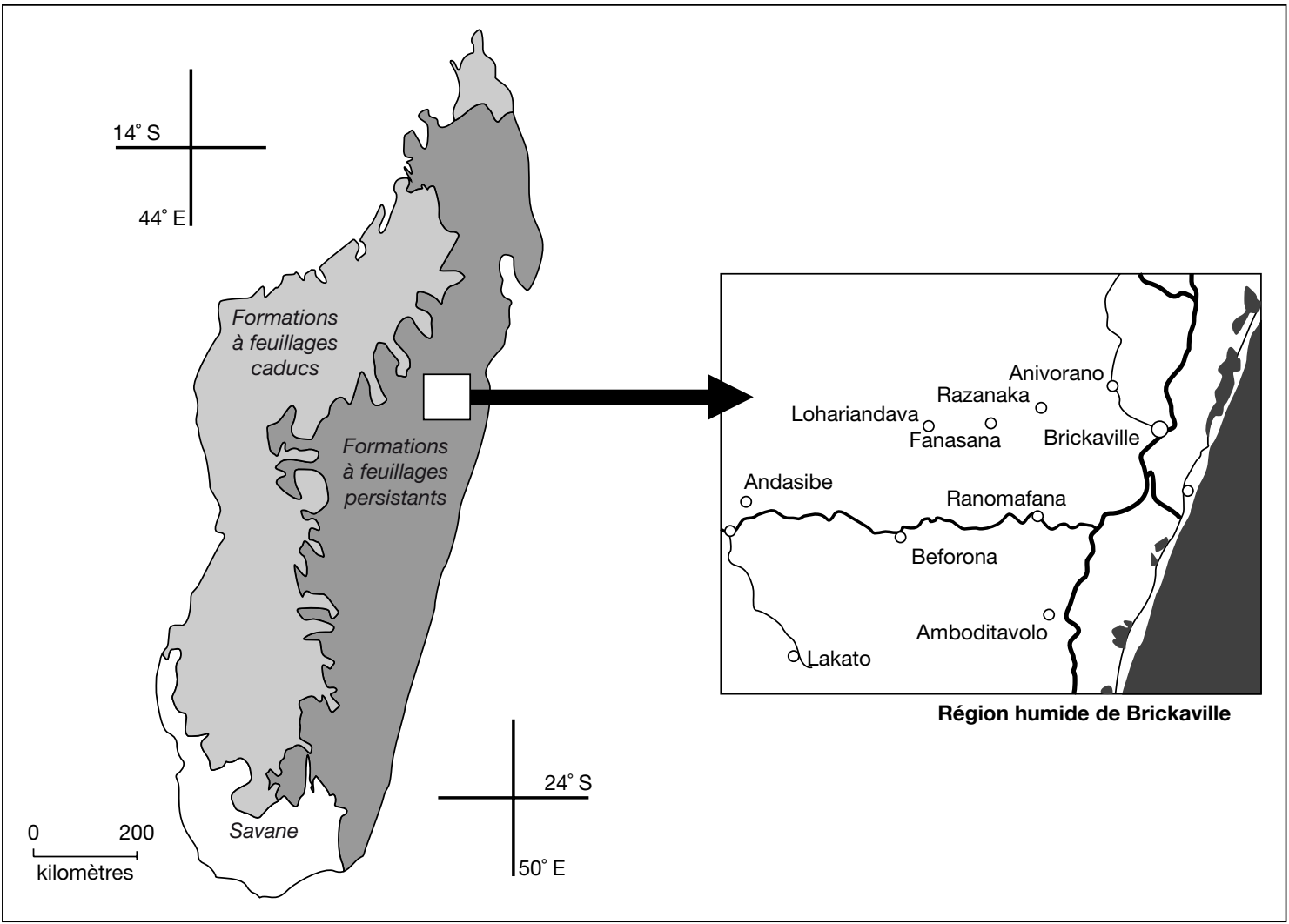

Figure 2. Lieux de récolte des ignames cultivées de la région de Brickaville (côte est de Madagascar).

Figure 2. Location of the study sites (East coast of Madagascar).

Tableau 1. Les ignames récoltées dans les régions de la côte est de Madagascar : identification, coordonnées géographiques, date et lieu de récolte.

Table 1. Yams harvested in the Eastern region of Madagascar.

\begin{tabular}{|c|c|c|c|c|c|c|}
\hline \multirow{2}{*}{$\begin{array}{l}\text { Numéro } \\
\text { MT } 229\end{array}$} & \multirow{2}{*}{$\begin{array}{l}\text { Nom scientifique } \\
\text { D. alata }\end{array}$} & \multirow{2}{*}{$\begin{array}{l}\text { Nom vernaculaire } \\
\text { ovilalaina }\end{array}$} & \multicolumn{2}{|c|}{$\begin{array}{l}\text { Coordonnées } \\
\text { géographiques }\end{array}$} & \multirow{2}{*}{$\begin{array}{l}\text { Date de récolte } \\
\text { 06/05/2008 }\end{array}$} & \multirow{2}{*}{$\begin{array}{l}\text { Lieu de récolte } \\
\text { Ranomafana }\end{array}$} \\
\hline & & & $18^{\circ} 58^{\prime} 17,3^{\prime \prime}$ & $48^{\circ} 45^{\prime} 39,2^{\prime \prime}$ & & \\
\hline MT 230 & D. alata & ovibe & $18^{\circ} 58^{\prime} 17,3^{\prime \prime}$ & $48^{\circ} 45^{\prime} 39,2^{\prime \prime}$ & 06/05/2008 & Ranomafana \\
\hline MT 237 & D. esculenta & mavondro & $18^{\circ} 58^{\prime} 17,3^{\prime \prime}$ & $48^{\circ} 45^{\prime} 39,2^{\prime \prime}$ & 06/05/2008 & Ranomafana \\
\hline MT 247 & D. alata & ovifotsy & $19^{\circ} 04^{\prime} 30,1$ & $48^{\circ} 54^{\prime} 47,2^{\prime \prime}$ & 09/05/2008 & Amboditavolo \\
\hline MT 253 & D. alata & ovibe & $18^{\circ} 50^{\prime} 27,6^{\prime \prime}$ & $47^{\circ} 33^{\prime} 34,0^{\prime \prime}$ & 22/05/2008 & Loharindava \\
\hline MT 255 & D. alata & ovilalaina & $18^{\circ} 50^{\prime} 27,6^{\prime \prime}$ & $47^{\circ} 33^{\prime} 34,0^{\prime \prime}$ & $22 / 05 / 2008$ & Loharindava \\
\hline MT 256 & D. esculenta & mavondro & $18^{\circ} 50^{\prime} 27,6^{\prime \prime}$ & $47^{\circ} 33^{\prime} 34,0^{\prime \prime}$ & 22/05/2008 & Loharindava \\
\hline MT 262 & D. alata & ovifotsy & $18^{\circ} 51^{\prime} 56,6^{\prime \prime}$ & $48^{\circ} 46^{\prime} 43,9^{\prime \prime}$ & 25/05/2008 & Fanasana \\
\hline MT 263 & D. alata & ovilalaina & $18^{\circ} 51^{\prime} 22,9^{\prime \prime}$ & $48^{\circ} 47^{\prime} 46,8^{\prime \prime}$ & 26/05/2008 & Fanasana \\
\hline MT 264 & D. esculenta & mavondro & $18^{\circ} 48^{\prime} 32,6^{\prime \prime}$ & $48^{\circ} 48^{\prime} 54,8^{\prime \prime}$ & $27 / 05 / 2008$ & Fanasana \\
\hline MT 269 & D. alata & ovifotsy & $18^{\circ} 47^{\prime} 54,4^{\prime \prime}$ & $48^{\circ} 52^{\prime} 02,7^{\prime \prime}$ & 29/05/2008 & Beforona \\
\hline MT 274 & D. alata & ovibe & $18^{\circ} 43^{\prime} 07,1^{\prime \prime}$ & $48^{\circ} 59^{\prime} 58,0^{\prime \prime}$ & 30/05/2008 & Anivorano \\
\hline MT 277 & D. esculenta & mavondro & $17^{\circ} 31^{\prime} 14,9^{\prime \prime}$ & $49^{\circ} 10^{\prime} 57,7^{\prime \prime}$ & 19/06/2008 & Lakato \\
\hline MT 281 & D. alata & ovilalaina & $19^{\circ} 04^{\prime} 30,1$ & $48^{\circ} 54^{\prime} 47,2^{\prime \prime}$ & 09/05/2008 & Amboditavolo \\
\hline
\end{tabular}

D. alata : Dioscorea alata ; D. esculenta : Dioscorea esculenta. 


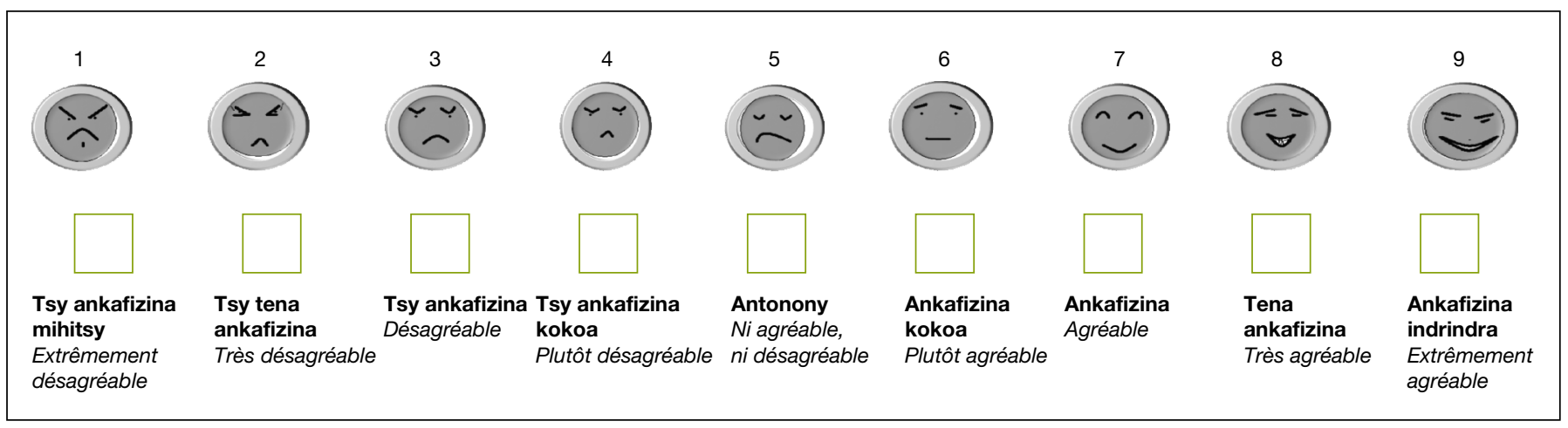

Figure 3. Échelle d'appréciation des ignames dans un test hédonique.

Figure 3. Appreciation scales in hedonic test.

Le climat de la région est de type perhumide chaud (pluviosité annuelle de plus de $2500 \mathrm{~mm}$ et une saison sèche peu marquée). Cette région est caractérisée par la présence de collines ne dépassant pas 200 mètres d'altitude, aux pentes plus ou moins fortes, où la forêt a disparu pour faire place à des jachères (ou savoka) herbacées à Aframomum angustifolium, puis arbustives à Ravenala madagascariensis (Humbert et Cours Darne, 1965).

\section{Préparation des échantillons}

Après la récolte, les tubercules n'ayant subi ni dommage ni attaque par les insectes, sont entreposés sur une plate-forme située à $1 \mathrm{~m}$ au-dessus du sol. La température de la chambre de conservation des tubercules frais varie entre 26 et $29{ }^{\circ} \mathrm{C}$ (Hygrothermograph, Model NS II-Q, Sato Keiryoki MFG Co. Ltd, Japan). Pour chaque variété, 5 à $7 \mathrm{~kg}$ de tubercules ont été lavés, épluchés, coupés en morceaux, trempés dans l'eau (P/V:1/1) puis cuits pendant 30 à $45 \mathrm{~min}$. Les échantillons ont été présentés tièdes aux consommateurs codés par un nombre aléatoire à trois chiffres.

\section{Test hédonique}

\section{Panel}

Le panel était constitué de six groupes de consommateurs dont un tiers provenait de la région de Brickaville (18 ${ }^{\circ} 48^{\prime} \mathrm{S}$ et $49^{\circ} 04^{\prime} \mathrm{E}$ ), un tiers de la commune urbaine d'Antananarivo $\left(18^{\circ} 52^{\prime} \mathrm{S}\right.$ et $\left.47^{\circ} 31^{\prime} \mathrm{E}\right)$ et un tiers de la commune rurale de Talata (18 $46^{\prime} \mathrm{S}$ et $47^{\circ} 34^{\prime} \mathrm{E}$ ) située à 25 kilomètres au nord-ouest d'Antananarivo. Pour chaque région, une moitié des groupes était constituée d'adultes $(n=60$, âges moyens \pm std : $48 \pm 2,35$ pour Brickaville, $41 \pm 1,65$ pour Antanarivo, et 44,5 $\pm 1,35$ pour Talata) et une moitié d'enfants d'âge scolaire ( $n=60$, âges moyens respectifs : $14,5 \pm 1,35, \quad 14,5 \pm 0,5, \quad 14 \pm 0,5$, pour Brickaville, Antananarivo et Talata). Une répartition équilibrée des deux sexes a été respectée au sein de chaque groupe.

\section{Procédure}

Le test hédonique a été réalisé dans les mêmes conditions pour les trois localités. Les consommateurs étaient convoqués entre 14 h 30 et 17 h 30 pour contrôler leur état de faim. Le test se déroulait dans une grande pièce par groupes de 20 consommateurs. Les consommateurs recevaient comme consigne de goûter les échantillons d'ignames un par un et d'indiquer leur appréciation globale sur une échelle hédonique de 9 points allant de "extrêmement désagréable " à

\section{Tableau 2. Définition des descripteurs d'ignames cuits utilisés lors des tests sensoriels.}

Table 2. Definition of the descriptors used to characterize cooked yams during the sensory tests.

\begin{tabular}{lll}
\hline Descripteurs & Échelle & Définition \\
\hline Régularité forme/couleur & 1 : irrégulière & Qui ne présente aucune variation de forme/couleur dans son étendue \\
& $7:$ très régulière & \\
Intensité du goût sucré & $1:$ pas du tout sucré & Degré de perception de la saveur sucrée \\
& $7:$ extrêmement sucré & \\
Couleur & $1:$ très foncée & Sensation perçue à l'œil nu \\
& $7:$ très claire & \\
Fermeté & $1:$ molle & État de ce qui offre une certaine résistance \\
& $7:$ très dure & État de ce qui est chargé d'eau \\
Humidité & $1:$ assez sec & \\
& $7:$ très humide & État de ce qui se joint étroitement à la surface des dents, \\
Adhérence & $1:$ pas collant & lors de la mastication de l'aliment \\
& 7 très collant & Capacité à émietter les tubercules et à les faire descendre \\
Capacité à avaler & $1:$ très difficile & dans le gosier
\end{tabular}


" extrêmement agréable". L'échelle était étiquetée en malgache et illustrée par des figures afin de faciliter la compréhension des termes (figure 3).

\section{Test descriptif}

Panel

Le panel était constitué de 9 hommes et 9 femmes, tous étudiants en sciences de l'alimentation, de 25 ans d'âge moyen pour les hommes, et de 24 ans pour les femmes.

\section{Entraînement}

Douze séances de formation ont été organisées sur une période de 6 semaines. Au cours des cinq premières séances, les panélistes décrivaient les tubercules en utilisant autant de termes que possible en langue française. Les termes générés ont été compilés pour former une liste préliminaire. Cette liste préliminaire a été utilisée pour décrire les tubercules sur une échelle de 7 points. Une analyse en composantes principales (ACP) a permis une réduction des termes afin d'obtenir la liste définitive (norme ISO11035, 1994) (tableau 2). Cinq séances d'entrầnement ont ensuite été réalisées pour familiariser les juges avec cette liste de descripteurs et les échelles de 7 points correspondantes. Les deux dernières séances ont permis de vérifier la reproductibilité et le caractère consensuel des juges dans leur notation.

\section{Évaluation}

Deux sessions d'évaluation ont été organisées. Pour chaque session, les juges entraînés recevaient les quatre variétés d'ignames et pour chaque variété, ils ont évalué sur une échelle de 7 points l'intensité des descripteurs retenus dans la liste définitive. Pour limiter l'effet de report d'une variété à l'autre, les juges se rinçaient la bouche avec de l'eau entre deux échantillons. Les échantillons étaient présentés selon un ordre aléatoire différent pour chaque juge. Les évaluations sensorielles ont été effectuées dans un laboratoire muni de cabines, spécialement conçues pour l'évaluation des aliments (Lawless et Heymann, 1999).

\section{Analyses physico-chimiques}

Les caractéristiques physico-chimiques des tubercules (humidité, teneurs en macronutriments et en micronutriments, facteurs antinutritionnels) ont été déterminées suivant les méthodes décrites par mists (AACC, 2000).

\section{Traitements statistiques}

\section{Test hédonique}

Une analyse de la variance (Anova) à trois facteurs a été réalisée sur les notes d'appréciation globale avec "âge des consommateurs" et "origine géographique" comme facteurs emboîtés et "variétés" comme facteur croisé. Lorsqu'un effet significatif a été observé, une comparaison de moyennes utilisant le test de Newman-Keuls (SNK) a été réalisée avec un risque $\alpha=0,05$.

\section{Profil descriptif}

Une Anova à deux facteurs avec répétition et "variétés d'ignames " comme fac-
l'American Association of Cereal Che-

teur croisé a été effectuée pour chacun des descripteurs. Lorsqu'un effet significatif a été observé, une comparaison de moyennes utilisant le test de NewmanKeuls (SNK) a été réalisée avec un risque $\alpha=0,05$.

\section{Caractérisation}

des quatre variétés d'ignames

Une analyse factorielle multiple (AFM) (Escofier et Pages, 1993) a été effectuée (SPAD V 5.5) pour mettre en relation l'appréciation des consommateurs avec les caractéristiques sensorielles et physico-chimiques des quatre variétés d'ignames. Trois groupes de variables continues illustratives et un groupe de variables nominales illustratives ont été soumis à l'analyse. Les trois groupes de variables continues incluaient les notes moyennes d'appréciation des ignames

\section{Tableau 3. Composition chimique des quatre variétés de tubercules d'igname cuits.}

Table 3. Chemical composition of four varieties of cooked yams (after peeling).

\begin{tabular}{lccrr}
\hline & D. esculenta & $\begin{array}{l}\text { D. alata } \\
\text { ovilalaina }\end{array}$ & D. ovifotsy & D. ovibe \\
\hline Humidité (g/100 g MS) & 76,1 & 77,9 & 77,8 & 79,4 \\
Matières grasses (g/100 g MS) & 0,3 & 0,4 & 0,5 & 0,3 \\
Protéines totales (g/100 g MS) & 4,2 & 4,1 & 3,7 & 1,8 \\
Glucides totaux (g/100 g MS) & 91,1 & 92,4 & 91,3 & 90,8 \\
Amidon (g/100 g MS) & 71,1 & 75,8 & 71,3 & 70,2 \\
Fibres (g/100 g MS) & 12,4 & 10,6 & 11,1 & 10,4 \\
Cendres (mg/100 g MS) & 3,0 & 1,3 & 2,8 & 2,8 \\
Potassium (mg/100 g MS) & 1540 & 910 & 1380 & 640 \\
Sodium (mg/100 g MS) & 20 & 10 & 10 & 5 \\
Calories (Cal) & 384,7 & 390,0 & 385,1 & 373,8 \\
\hline
\end{tabular}

Les valeurs correspondant à une moyenne effectuée sur trois répétitions (valeurs après épluchage). MS : matière sèche ; D. : Dioscorea.

\section{Tableau 4. Note moyenne d'appréciation des quatre variétés d'ignames en fonction de l'âge et de la région d'origine des consommateurs.}

Table 4. Average appreciation score as a function of consumer age and region of origin.

\begin{tabular}{lcccccc}
\hline & \multicolumn{2}{c}{ Talata } & \multicolumn{2}{c}{ Antannarivo } & \multicolumn{2}{c}{ Brickaville } \\
& Enfants & Adultes & Enfants & Adultes & Enfants & Adultes \\
\cline { 2 - 7 } D. alata ovibe & 5,8 & 6,48 & 4,83 & 5,81 & 6,4 & 6,43 \\
D. alata ovilalaina & 7,03 & 7,35 & 6,8 & 6,91 & 6,76 & 7,28 \\
D. alata ovilavafotsy & 5,23 & 5,65 & 4,51 & 4,9 & 5,83 & 5,8 \\
D. esculenta & 7,46 & 7,75 & 7,46 & 7,58 & 7,58 & 7,7 \\
\hline
\end{tabular}

D. : Dioscorea. 
obtenues par les six groupes de consommateurs (hédonique), les notes moyennes obtenues pour les descripteurs sensoriels discriminants (c'est-à-dire pour lesquels un effet produit a été observé dans l'Anova) et les paramètres physicochimiques moyennés sur trois répétitions (tableau 3). Le groupe de variables nominales était constitué par des paramètres nutritionnels obtenus par le criblage phytochimique et codés en présence absence.

\section{Résultats et discussion}

\section{Test hédonique}

Dans l'ensemble, les notes moyennes d'appréciation des ignames sont relativement élevées (tableau 4). Il semble donc qu'en l'absence d'information extérieure, il n'y ait pas de rejet des tubercules d'ignames. Toutefois, les résultats de l'Anova ont montré un effet significatif de l'âge des participants $\mathrm{F}(1,354)=93,79, p<0,0001$, de leur région d'origine $\mathrm{F}(2,354)=124$, $p<0,0001$, de la variété d'ignames $\mathrm{F}$ $(3,1062)=975,94, \quad p<0,0001$ et d'une interaction entre ces trois facteurs. La comparaison des moyennes indique que parmi les tubercules étudiés, $D$. esculenta est la plus appréciée, avec une note moyenne de 7,59. Les formes ovilalaina, ovibe, ovifotsy de D. alata sont appréciées d'une manière décroissante avec des notes moyennes de 7,$02 ; 5,96 ; 5,32$. Globalement, les adultes $(\mathrm{m}=6,64)$ ont davantage apprécié les ignames que les enfants d'âge scolaire $(m=6,31)$. Cette plus grande appréciation des ignames par les consommateurs adultes est avérée pour les quatre variétés d'ignames (figure 4A) mais seulement pour deux régions: Talata et Antananarivo (figure $4 B$ ). Concernant la région d'origine, les consommateurs de Brickaville $(\mathrm{m}=6,73)$ ont tendance à apprécier davantage les ignames que ceux de la commune rurale de Talata $(\mathrm{m}=6,59)$ qui eux-mêmes les apprécient davantage que ceux de Tananarive $(6,10)$. Cette différence entre les trois régions n'est toutefois significative que pour les deux formes d'ignames les moins appréciées (ovibe et ovifotsy), aucune différence n'étant observée pour la variété la plus appréciée $(D$. esculenta). Pour la forme ovilalaina, seule une différence entre Talata et Tananarive est observée (figure 4C).

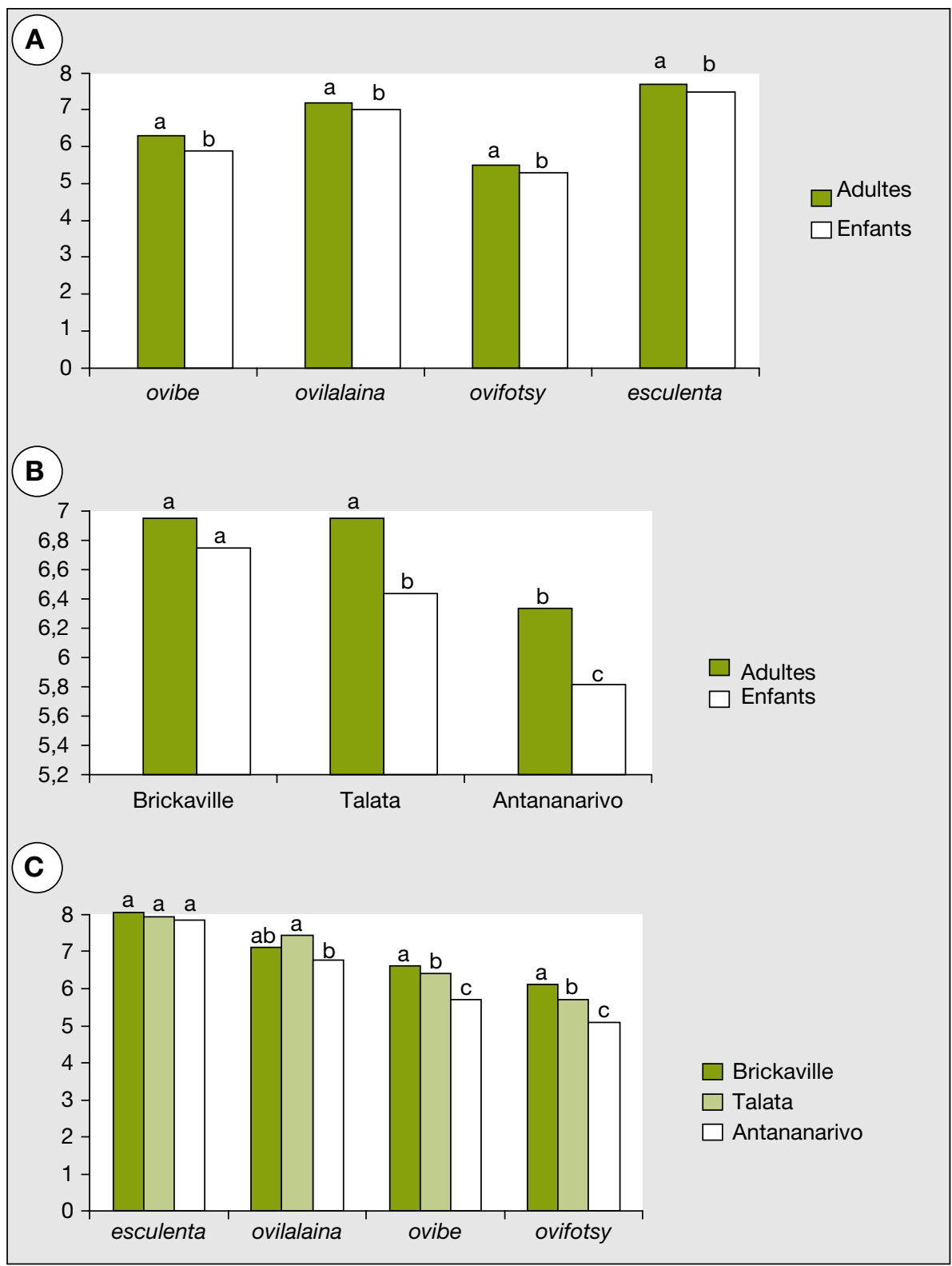

Figure 4. Note moyenne d'appréciation des ignames.

Figure 4. Average appreciation scores as a function of consumer age and yam variety, consumer age and region of origin, and consumer region of origin and yam variety.

A) selon l'âge des consommateurs et de la variété d'igname ; B) selon l'âge des consommateurs et leur région d'origine; C) selon la région d'origine des consommateurs et de la variété d'igname.

Les lettres indiquent le résultat du test de comparaison de moyenne (SNK, $\alpha=0,05)$ : deux lettres différentes indiquent des notes significativement différentes.

\section{Test descriptif}

Les résultats de l'Anova (tableau 5) ont montré un effet significatif au seuil $\alpha=0,05$ de la variété d'igname sur tous les descripteurs, sauf les descripteurs "humide" et "difficulté à avaler". Ces deux descripteurs ne permettant pas de discriminer entre les quatre varié- tés d'igname, ils ne seront pas conservés pour la suite des analyses. Un effet répétition a été observé pour le descripteur "régularité de la couleur ", mais cet effet n'est pas problématique dans la mesure où l'interaction " répétition*variété " n'est pas significative. Une interaction " répétition*variété" a été observée pour les descripteurs "régularité de la forme " et 
"couleur". Cette interaction reflète le manque d'homogénéité des échantillons pour ces deux descripteurs qui seront donc utilisés avec prudence dans la suite des analyses. Le test de comparaison de moyennes effectuées sur les descripteurs discriminants (tableau 5) indique que :

- D. esculenta (Lour.) présente des tubercules de forme assez régulière, de couleurs très claires, de saveur sucrée, de textures plus ou moins collantes en bouche, légèrement molles au toucher ; - La forme ovilalaina présente des tubercules de forme variable, de couleur assez régulière et plutôt foncée, de saveur assez sucrée, de textures assez collantes en bouche, et légèrement molles au toucher ;

- La forme ovibe présente des tubercules de forme irrégulière, de couleur foncée, de saveur légèrement sucrée, de textures légèrement collantes en bouche et légèrement molles au toucher ;

- La forme ovifotsy présente des tubercules de forme peu régulière, de couleur régulière, de saveur légèrement sucrée, et de textures plus ou moins collantes en bouche, légèrement molles au toucher.

\section{Caractérisation des quatre variétés d'ignames}

Les figures $5 A$ et $5 B$ représentent respectivement la projection des variables et des variétés d'ignames sur les deux premières composantes principales de l'AFM. La première composante principale explique $52 \%$ de la variance totale. Elle oppose les formes ovibe et ovifotsy, décrites comme étant fibreuses, humides, avec des traces d'alcaloïdes et de saponines, aux tubercules ovilalaina et D. esculenta décrits comme étant sucrés et faciles à avaler avec la présence de leucoanthocyanes. Ces deux dernières variétés comportent davantage de glucides, d'amidon et fournissent plus de calories que les deux autres et sont davantage appréciées par l'ensemble des consommateurs.
La composante principale 2 explique $29 \%$ de la variance totale. Elle permet essentiellement de différencier les formes ovibe et ovifotsy. La première se caractérise par des teneurs en cendres, matières grasses, sodium, et potassium plus élevées que la seconde. Celle-ci est décrite comme plus ferme avec une plus grande régularité de forme et de couleur que la forme ovibe.

Un calcul des coefficients de corrélation de Pearson entre les notes hédoniques données par les six groupes de consommateurs et les mesures sensorielles et physico-chimiques (tableau o) montre que, globalement, l'appréciation des ignames est corrélée négativement avec le taux d'humidité et de fibres et positivement avec le taux d'amidon, de glucides, l'apport de calories, le caractère sucré et la facilité à avaler. Comparativement aux autres groupes de consommateurs les consommateurs enfants d'âge scolaire de Talata et d'Antananarivo semblent plus sensibles au caractère sucré des ignames qu'aux autres facteurs.

\section{Tableau 5. Résultats de l'Anova et du test de comparaison de moyenne (SNK, $\boldsymbol{\alpha}=\mathbf{0 , 0 5}$ ) pour le profil}

sensoriel.

Table 5. ANOVA and paired comparison test (SNK, $\alpha=0.05)$ results for sensory profiles.

\begin{tabular}{|c|c|c|c|c|c|c|c|}
\hline Descripteurs & Répétition & Produit & Répétition*produit & D. ovibe & D. ovilalaina & D. ovifotsy & D. esculenta \\
\hline Régularité de la forme & ns & $p<0,0001$ & $p<0,05$ & $2,0 d$ & $2,6 c$ & $3,7 b$ & $5,1 a$ \\
\hline Régularité de la couleur & $p<0,001$ & $p<0,0001$ & ns & $3,9 b$ & $5,6 a$ & $5,7 a$ & $5,4 a$ \\
\hline Couleur & ns & $p<0,0001$ & $p<0,001$ & $4,5 b$ & $2,5 d$ & $6,4 a$ & $4,0 c$ \\
\hline Sucré & ns & $p<0,0001$ & ns & $2,5 b$ & $4,4 a$ & $2,8 b$ & $4,8 a$ \\
\hline Adhérence & ns & $p<0,0001$ & ns & $3,2 b$ & $3,6 a$ & $1,9 d$ & $2,3 c$ \\
\hline Ferme & ns & $p<0,0001$ & ns & $1,7 c$ & $2,0 b$ & $2,4 a$ & $2,4 a$ \\
\hline
\end{tabular}

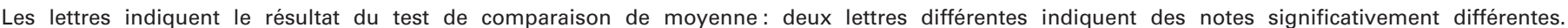
$D$. : Dioscorea; ns : non significatif.

\section{Tableau 6. Coefficients de corrélation de Pearson entre mesures hédoniques, test descriptif et composition chimique.}

Table 6. Pearson correlation coefficient between hedonic scores, sensory descriptors and chemical composition.

\begin{tabular}{|c|c|c|c|c|c|c|c|}
\hline & Humidité & Glucide & Amidon & Fibre & Calories & Sucré & $\begin{array}{l}\text { Facilité } \\
\text { à avaler }\end{array}$ \\
\hline Enfants Talata & $-0,89$ & 0,87 & 0,87 & $-0,87$ & 0,72 & 0,95 & 0,84 \\
\hline Adultes Talata & $-0,93$ & 0,92 & 0,93 & $-0,79$ & 0,81 & 0,89 & 0,87 \\
\hline Enfants Antananarivo & $-0,86$ & 0,83 & 0,80 & $-0,92$ & 0,64 & 0,98 & 0,77 \\
\hline Adultes Antananarivo & $-0,94$ & 0,92 & 0,92 & $-0,81$ & 0,80 & 0,90 & 0,84 \\
\hline Enfants Brickaville & $-0,99$ & 0,97 & 0,90 & $-0,71$ & 0,85 & 0,84 & 0,71 \\
\hline Adultes Brickaville & $-0,93$ & 0,91 & 0,91 & $-0,82$ & 0,78 & 0,91 & 0,85 \\
\hline
\end{tabular}

Seuls les coefficients supérieurs à $0,60(p<0,0001)$ sont indiqués 
(A)

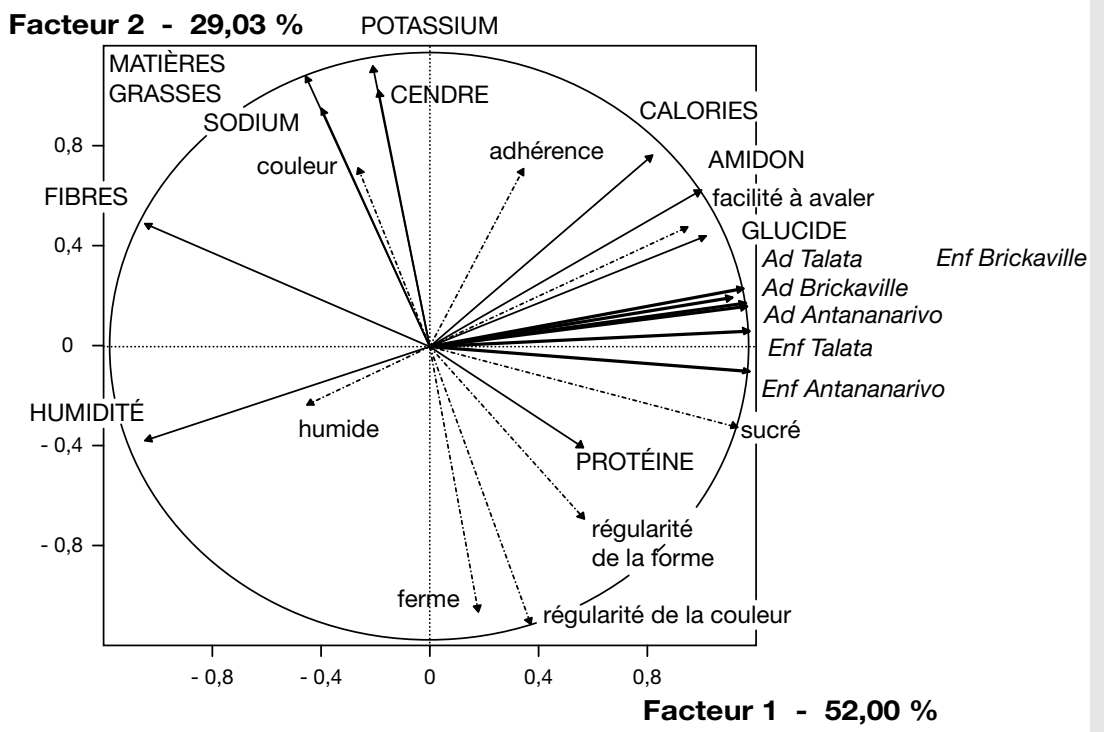

(B)

Facteur 2 - 29,03\%

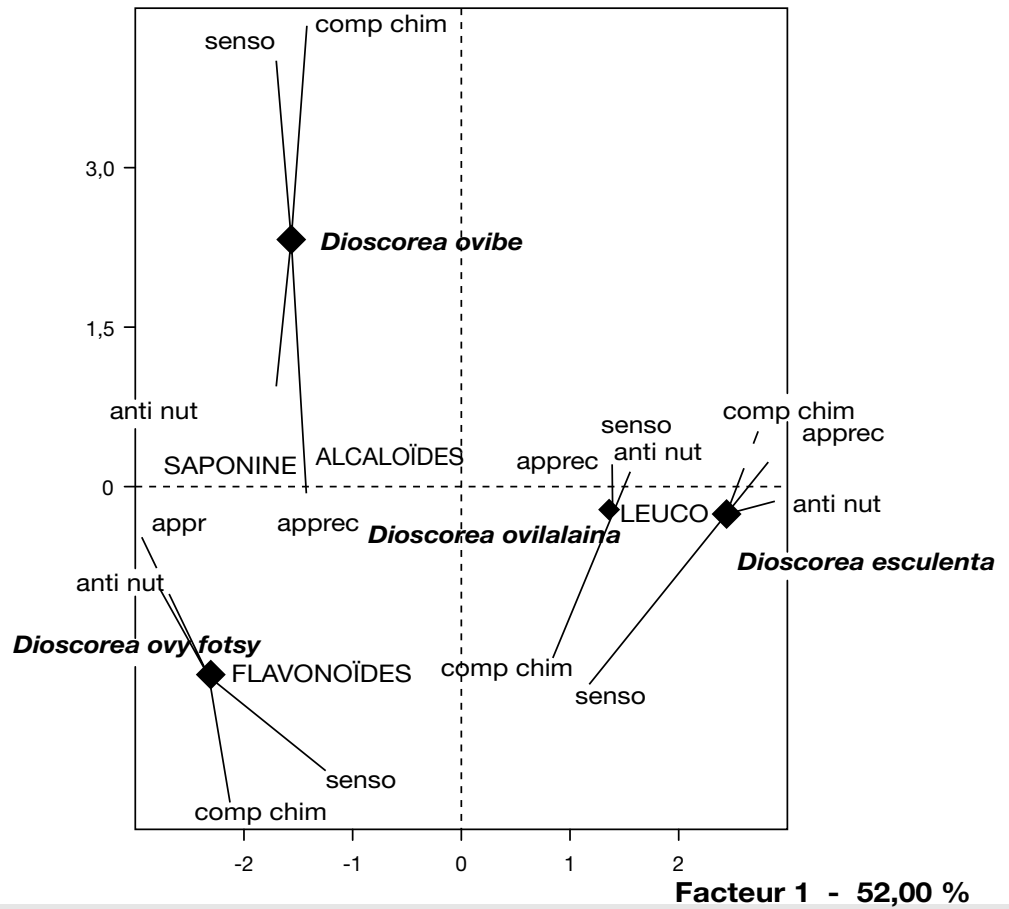

Figure 5. Analyse factorielle multiple (AFM) des quatre variétés d'ignames

Figure 5. Multiple Factorial Analysis (MFA) of the four yam varieties.

A) cercle de corrélation ; B) projections des échantillons d'ignames.

Ad : adultes ; enf : enfants ; senso : données sensorielles ; comp chim : données chimiques ; anti nut : données antinutritionnelles; apprec : appréciation par les consommateurs.

\section{Conclusion}

Cette étude constitue une première caractérisation sensorielle des tubercules de D. esculenta, ainsi que des formes ovibe, ovilalaina, ovifotsy de l'espèce D. alata, les plus cultivées de Madagascar. L'espèce D. esculenta est celle qui a la préférence des consommateurs malgaches, quels que soient leur âge et leur origine. La saveur sucrée et la fermeté constituent les facteurs d'appréciation de cette variété par les consommateurs. Cette étude a permis d'apporter une base scientifique pour la valorisation des ignames cultivées de Madagascar. Les résultats obtenus ont permis d'identifier une espèce intéressante aux yeux des consommateurs malgaches, et de faire progresser les connaissances dans le domaine alimentaire des ignames malgaches. Les variétés de D. alata de Madagascar semblent avoir les mêmes propriétés organoleptiques que celles des variétés cultivées d'Afrique occidentale $(D$. alata, D. rotundata Poir.). Les caractéristiques des ignames influant sur leur acceptabilité sont l'aspect général, la couleur, la saveur sucrée et la texture de la chair.

\section{Remerciements}

Le programme de recherche sur les ignames cultivées de Madagascar a été financé par le Projet de la Coopération pour la recherche universitaire et scientifique (CORUS 6020). Nous remercions tout particulièrement le Laboratoire d'analyse sensorielle d'Antananarivo (LAS) pour tous les travaux d'évaluation sensorielle, les populations des communes rurales de Talata, et de Brickaville pour les tests consommateurs ainsi que les étudiants en sciences des aliments de la faculté des sciences et de l'Ecole supérieure des sciences agronomiques, de l'université d'Antananarivo, en tant que membres du jury expert.

\section{Références}

AACC. Approved Methods of the American Association of Cereal Chemists. 8th edition. St Paul (M.N., USA) : AACC, 2000.

Akissoe HN, Hounhouigan DJ, Bricas N, Vernier $\mathrm{P}$, Nago CM, Olorunga OA. Physical, chemical and sensory evaluation of dried yam (Dioscorea rotundata) tubers, flour and "amala", a flour derived product. Tropical Sciences 2001; 41 : 151-5. 
Amani NG. Propriétés physico-chimiques et moléculaires des amidons d'igname (Dioscorea spp.) cultivées en Côte d'Ivoire. Relation avec la stabilité des gels aux traitements technologiques. Thèse de doctorat d'état en Sciences, université d'Abobo Adjamé, Côte d'Ivoire, 2002.

Brunnschweiler J, Luethi D, Handschin S, et al. Isolation, physico chemical characterization and application of Yam (Dioscorea spp.) starch as thickening and gelling agent. Starch/Stârkr $2005 ; 57: 107-17$

Dumont R, Dansi A, Vernier P, Zoundjihekpon J. Biodiversité et domestication des ignames en Afrique de l'Ouest: pratiques traditionnelles conduisant à Dioscorea rotundata. Collection Repères. Montpellier : Cirad, 2005.

Escofier B, Pages J. Analyse factorielle simple et multiple. Paris : Dunod, 1993.

Humbert H, Cours Darne G. Notice de la carte Madagascar. Trav. Sect. Sc. Tech. Pondichéry: Institut français de Pondichéry, 1965.

Jeannoda V, Jeannoda VH, Hladik A, et al. Les ignames de Madagascar: Diversité, utilisations et perceptions. Hommes et Plantes 2003; 47: $10-23$.
Jeannoda VH, Razanamparany JL, Rajaonah MT, et al. Les ignames (Dioscorea spp.) de Madagascar: espèces endémiques et formes introduites; diversité, perception, valeur nutritionnelle et systèmes de gestion durable. Rev Ecol (Terre Vie) 2007; 62 : 191-207.

Lawless HT, Heymann H. Sensory evaluation of food: principles and practices. New York : Kluwer Academic/Plenum Publishers, 1999.

Nindjin C, Brunnschweiler J, Konan G, et al. Plaisir du consommateur et caractérisation sensorielle et instrumentale de la qualité des ignames (Dioscorea spp.) Bioterre, Revue Internationale des Sciences de la Vie et de la terre 2002; 9: 336-343.

Nindjin C, Otokore DA, Hauser S, et al. Determination of relevant sensory properties of pounded yams (Dioscorea spp.) using a locally-based descriptive analysis methodology. Food Quality and Preference 2006; 18 : 450-9.

Onayemi O. Sensory texture profile of African foods made from yam and cassava. Journal of Texture Studies $1985 ; 16: 263-9$.

Onayemi O, Idowu A. Physical and chemical changes in traditionally stored yam tubers (Dioscorea rotundata Poir and Dioscorea cayenensis Lam.). Journal of Agricultural Food chemistry $1988 ; 36$ : 588-91.
Perrier de la Bâthie H. Ignames cultivées ou sauvages de Madagascar. Rev Bot Appl Agron Colon $1925 ; 5: 417-22$.

Razanamparany JL, Ralaiarison GD, Jeannoda $\mathrm{VH}$, et al. Potentialités nutritionnelles et alimentaires des ignames malgaches. Yaoundé (Cameroun) : International Meeting Food Africa, 2003.

Treche S, Mbome Lape L, Agbor Egbe T. Variation de la valeur nutritionnelle au cours de la préparation de produits séchés à partir d'ignames cultivées au Cameroun (Dioscorea dumetorum et $D$. rotundata). Revue Sciences et Techniques $1984 ; 4: 77-92$

Vernier P, Dossou RA, Letourmy P. Influence de la fertilisation chimique sur les qualités organoleptiques de I'igname. Cah Agric $2000 ; 9$ : 131-4.

Vernier P, Dossou RA. Exemple de sédentarisation de la culture des ignames: cas des Kokoro au Bénin. Agronomie africaine 2003 ; 15 : 187-96.

Wilkin $\mathrm{P}$, Schols $\mathrm{P}$, Chase MW, et al. A plastid gene phylogeny of the yam genus Dioscorea $L$. (Dioscoreaceae): roots, fruits of Madagascar. Systematic Botany 2005 ; 30 : 736-49. 